\title{
Magnetic Resonance Imaging of the Bowel: Today and Tomorrow
}

\section{Magnetresonanztomografie des Darms: Altbewährtes und Innovatives}

Authors

Affiliation
S. Kinner, M. L. Hahnemann, M. Forsting, T. C. Lauenstein

Department of Diagnostic and Interventional Radiology and Neuroradiology, University Hospital Essen, Germany

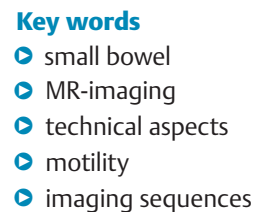

received $\quad 4.3 .2014$

accepted $\quad 11.9 .2014$

Bibliography

DOI http://dx.doi.org/

10.1055/s-0034-1385453

Published online: 2015

Fortschr Röntgenstr 2015; 187:

160-167 @ Georg Thieme

Verlag KG Stuttgart · New York .

ISSN 1438-9029

Correspondence

Frau Dr. Sonja Kinner

Institut für Radiologie,

Universitätsklinikum Essen

Hufelandstraße 55

45122 Essen

Germany

Tel.: ++ 49/02 01/7232311

Fax: ++ 49/02 01/7231563

Sonja.Kinner@uni-due.de

\section{Abstract \\ $\nabla$}

Magnetic resonance imaging of the small bowel has been feasible for more than 15 years. This review is meant to give an overview of typical techniques, sequences and indications. Furthermore, newly evaluated promising techniques are presented, which have an impact on the advance of MR imaging of the small and large bowel.

Key Points:

- T2-weighted sequences both with and without fat saturation and $\mathrm{T} 1$-weighted fat saturated sequences prior to and following intravenous injection of a gadoliniumbased contrast medium contitute the basics for bowel MR imaging.

- Newer MR applications, such as diffusionweighted imaging or contrast-enhaced dynamic sequences supply addtional information; they should thus be integrated in a regular sequence protocol für bowel MRI.

- Additional new modalities like motility imaging and PET/MRI have to be evaluated in future studies.

Citation Format:

- Kinner S, Hahnemann ML, Forsting M et al. Magnetic Resonance Imaging of the Bowel: Today and Tomorrow. Fortschr Röntgenstr 2015; 187: 160-167

\section{Zusammenfassung}

Die Möglichkeit zur Darstellung des Darmtrakts mittels Magnetresonanztomografie besteht bereits seit mehr als 15 Jahren. Dieser Übersichtsartikel soll einen Überblick über die typischen Techniken, Sequenzen und Indikationen geben. Zudem werden in den letzten Jahren neu evaluierte vielversprechende Techniken dargestellt, die einen weiteren Fortschritt in der MRT-Bildgebung des Dünn- und Dickdarms bieten.

\section{Introduction}

$\nabla$

Magnetic resonance imaging (MRI) has been used for examining the small and large intestines for over 15 years [1, 2]. During this period, new techniques and sequences have provided imaging with consistency and have ensured ongoing improvement and innovation.

This survey article provides a summary of time-tested principles of imaging and sequence protocols and the known and typical indications. It also presents new and future applications and techniques.

\section{Time-tested}

$\checkmark$

\section{Indications}

The typical indications for magnetic resonance imaging (MRI) of the bowel are inflammatory or tumorous changes [3]. MRI of the bowel, in particular the large intestine, was and is still discussed as a screening method for colorectal cancers, which are frequently treatable and even curable if detected at an early stage $[4,5]$. An article recently published by Graser et al. demonstrates that MR colonography at 3 Tesla detects colorectal adenomas $\geq 6 \mathrm{~mm}$ and advanced neoplasia with greater sensitivity and specificity, yet still remains inferior to colonoscopy when it comes to colorectal neoplasia [6].

A suspected diagnosis in the large intestine can be routinely confirmed through endoscopy and histological study. In cases of incomplete colonoscopy, e.g. when there are stenotic colon tumors or unfavorable anatomy as 

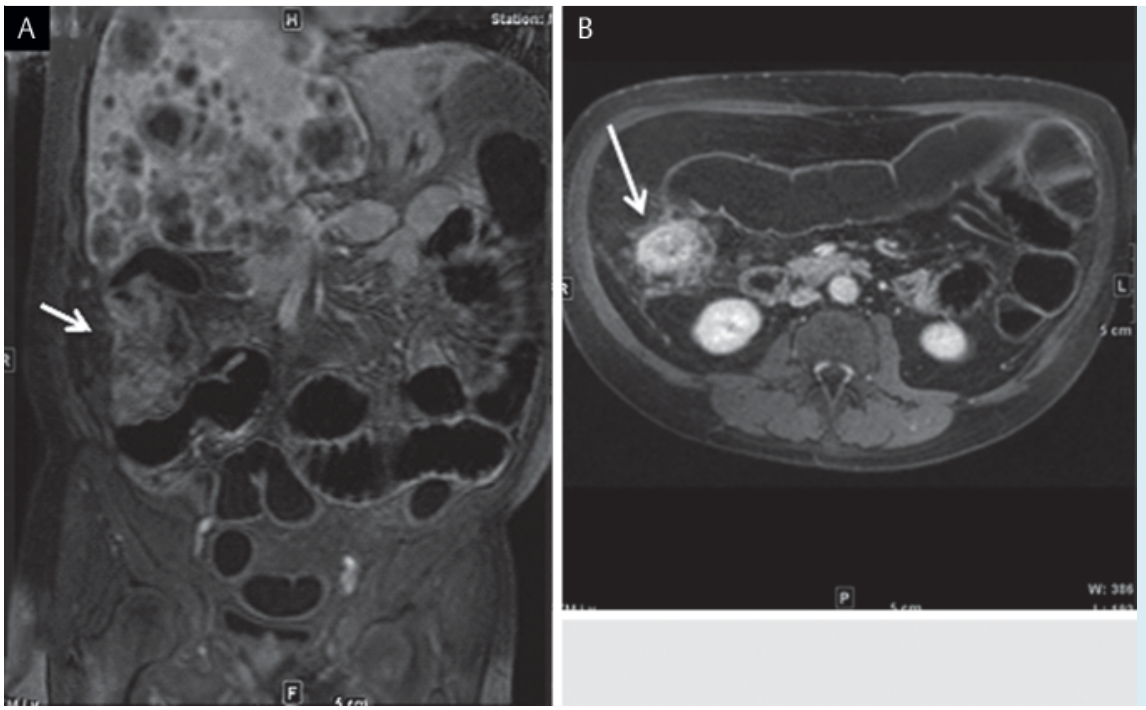

Fig. 166 year old male patient with histopathological confirmed diagnosis of a colonic cancer in the ascending colon (arrow in $\mathbf{A}$ coronal fat saturated VIBE sequence in arterial phase). MR colonography shows liver metastases in addition to the primary cancer and locoregional lymphangiosis (arrow in B fat saturated T1 TSE axial after contrast).

well as following infections, MRI makes it possible to evaluate sections of the colon more proximal [7]. For diseases of the small intestine, capsule endoscopy currently provides image-based morphological confirmation of a diagnosis, yet is still used on a limited basis because of its costliness. MRI of the small bowel constitutes a more cost-effective and very good diagnostic method for confirming or excluding diseases in this section of the bowel. MRI of the small intestine is performed primarily in cases of chronic inflammatory bowel disease in children and adults [8, 9]. The advantage of MRI over computed tomography is that it involves no exposure to radiation, which is particularly important given the young patient age and the necessity of repeated examinations for monitoring therapy. MRI has the advantage over endoscopy in that it can also examine extraintestinal areas, which is especially important when dealing with transmural inflammatory diseases such as Crohn's disease and when assessing local and remote metastases of intestinal tumors ( $\bullet$ Fig. 1). In addition, MRI of the small intestine is an observer-independent method as Schleder et al. demonstrated in an analysis of MR enterographies performed on patients with Crohn's disease [10].

\section{Examination prerequisites, protocol and sequences}

To achieve diagnostic image quality and to facilitate performing the selected sequences within one breathhold, the bowel should be examined at least on a 1.5 Tesla MRI machine. Thus far, higher field strengths have not been found to be superior in detecting polyps when used in MR colonography $[11,12]$ and have exhibited only minor advantages in diagnosing mucosal ulcerations in Crohn's patients [13]. Therefore, 1.5-Tesla scanners, which are the most commonly available in clinical settings, continue to be the standard for imaging. The distension of the cleansed and evacuated bowel is another prerequisite for optimal imaging. In recent years, various iso- and hyperosmolar liquids have been tested for use in distending the small intestine [14]. At our clinical practice, we use a mannitol and locus bean gum solution [15] prepared by our in-house pharmacy that is on par with other commercially available liquids [16-18]. While the question whether the contrast medium should be delivered to the intestinal tract orally (MR enterography) or via an already in place nasojejunal probe (MR enteroclysis) is widely debated, it has been the subject of only a few comparative studies. In their respective comparative studies, Masselli et al. and Negaard et al. found that significantly better distension was achieved with MR enteroclysis than with MR enterography [19, 20]. However, Negaard et al. were able to detect Crohn's-typical changes with high diagnostic accuracy and reproducibility using either method [20]. In their intraindividual comparison of MR enteroclysis and MR enterography, Schreyer et al. demonstrated that all pathologies were correctly detected with both MR methods [21]. They thus concluded already in 2004 that patientfriendly MR enterography has the potential to replace the conventional enteroclysis. Today, MR enterography is the preferred method at many centers given its greater simplicity, practicality and non-invasiveness, and in view of the radiation involved when inserting the nasojejunal probe for MR enteroclysis [22]. MRI of the bowel is initiated after approximately $1500 \mathrm{ml}$ of iso- to hyperosmolar fluid is ingested over approximately 45 minutes. At many centers, metoclopramide is used to promote gastric peristalsis and emptying. The colon is filled rectally with conventional tap water to provide contrast [23]. If rectal filling is not desired or necessary (e.g. if the primary focus is on imaging the small intestine) the colon can be sufficiently filled with the oral contrast medium by allowing a somewhat longer waiting time or by having the patient drink the liquid in intervals. In a comparative study of MR enterographies on patients with Crohn's disease, Friedrich et al. showed that the additional rectal contrast by means of water enema facilitated greater sensitivity and specificity particularly in the terminal ileum, ascending colon and rectum [24].

The classic examination protocol for examining the large and small intestine includes axial and coronal T1- and T2weighted sequences as well as the acquisition of dynamic contrast enhanced T1-weighted sequences [25]. To keep the motion-related artefacts to a minimum, the examination should be performed with the patient lying prone, since this reduces respiratory excursion, and an antispasmotic should be administered to suspend or significantly reduce intestinal motility. The appropriate antispasmotics are hyoscine butylbromide, (Buscopan, Boehringer Ingel- 

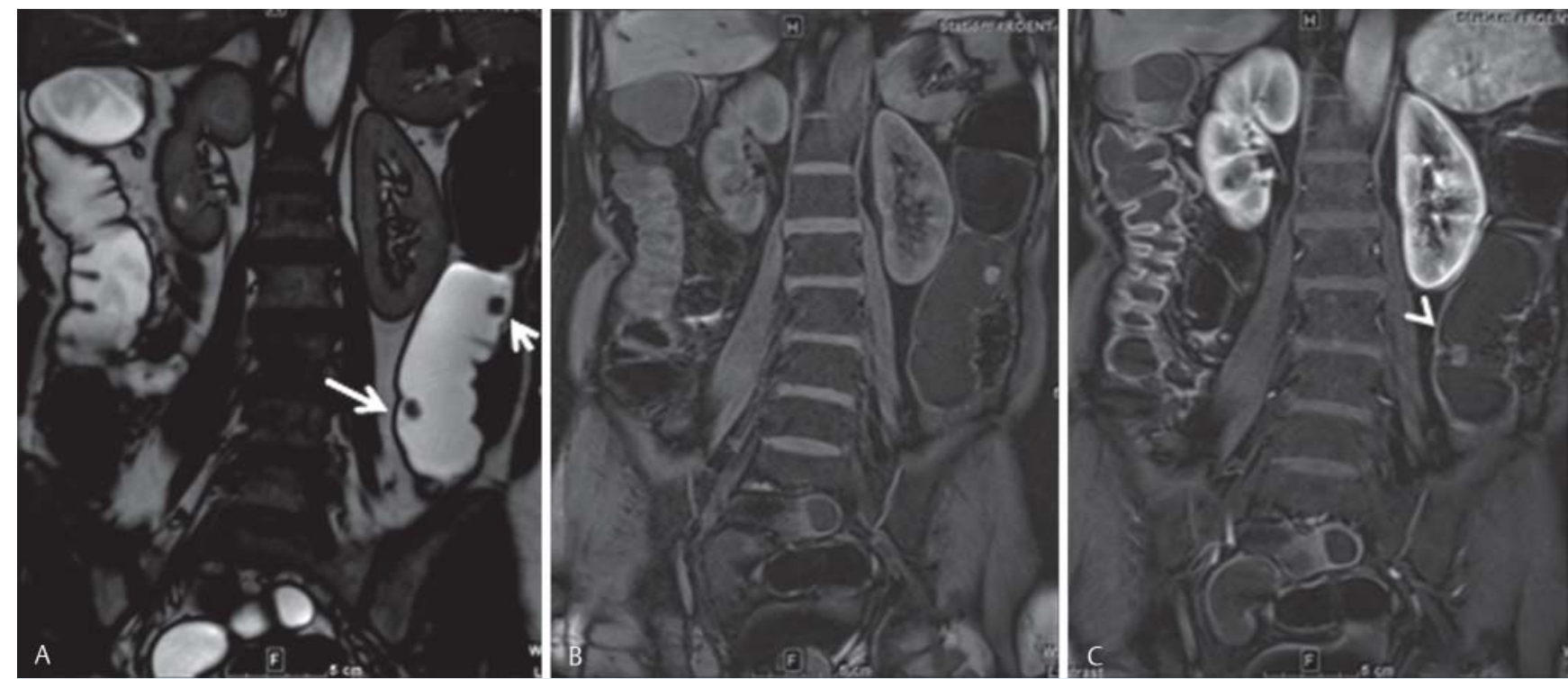

Fig. 2 MR colonography in a 46 year old female patient with anemia due to iron deficiency. Two lesions can be depicted in the TrueFISP images $\mathbf{A}$ in the descending colon (arrows). The proximal lesion is hyperintense in an unenhanced T1-weighted sequence $\mathbf{A}$ and cannot be depicted after con- trast $\mathbf{C}$ leading to the diagnosis of residual stool. The second lesion is only visible after contrast (arrow head): Subsequent colonoscopy confirmed a polyp. heim Pharma GmbH \& Co KG, Germany) and glucagon, with studies showing glucagon to induce a longer period of spasmolysis [26]. Intravenous administration results in a faster and more reliable onset of effect [27].

One of the relevant sequences is an axial or coronal T2weighted sequence (half-fourier single-shot turbo spin echo, HASTE with Siemens; single-shot fast spin echo, SSFSE with General Electric or ultra-fast spin echo, UFSE with Philips), which offers a good overview of the wall thickness of the intestinal segments as well as of any existing edema. A balanced Steady State Free Precession (bSSFP) sequence (True fast imaging with steady state precession, TrueFISP with Siemens; Fast Imaging Employing Steady-state Acquisition, FIESTA with General Electric or Balanced Fast Field Echo, bFFE with Philips) in coronal acquisition facilitates good clarification of mural pathologies such as ulcerations as well as extraintestinal changes such as mesenteric lymph nodes or vascular injections (comb signs) as signs of inflammatory changes. This sequence is not sensitive to movements and can thus be used as a basic sequence on patients with reduced compliance. In addition, coronal T1-weighted, fat-saturated rapid gradient echo sequences are performed for dynamic contrast-enhanced acquisition (volume-interpolated breath hold examination, VIBE with Siemens, liver acquisition with volume acquisition, LAVA with General Electric, enhanced T1 High Resolution Isotropic Volume Excitation, eTHRIVE with Philips). Finally, a fat-saturated T1 Turbo Spin Echo (TSE) sequence is performed in axial and coronal acquisition during the late phase following administration of contrast medium to allow the surrounding organs and soft tissues to be examined for pathologies once again. In their evaluation of the specified sequences, Schleder et al. concluded that a non-contrast T1-weighted sequences can be omitted from the MRI-supported diagnostic investigation of Crohn's disease [28]. However, this type of sequence plays an important role in the diagnostic investigation of the large intestine since it allows possible contrast-enhanced polyps to be differentiated from stool residue, which is already hyperintense in non-contrast sequences [29] as $\bullet$ Fig. 2 shows. By prompting subsequent endoscopic removal, polyp detection is important, since a polyp may result in a later intestinal tumor in the course of an adenoma-carcinoma sequence (see also - Fig. 1). Non-enhanced T1-sequences should therefore be part of in a conclusive bowel protocol. Fig. 3 provides an overview of a typical basic protocol for bowel diagnostics.

\section{Innovative \\ $\nabla$}

\section{Diffusion-weighted imaging}

Diffusion-weighted sequences were first used in the neuroradiological MR diagnostic investigation of strokes [30] as well as in the differentiation of brain tumors [31]. In the meantime, this technique has also been used for full body diagnostics, in particular to answer oncological clinical requests [32, 33]. Diffusion weighted imaging (DWI) essentially takes advantage of the limited diffusion (random thermal motion of the water molecules / Brownian molecular motion) caused by, for example, damaged membranes in infarcted areas. Limited diffusion is also present in inflamed or tumorous tissue. In tumors, the cells are more densely packed, resulting in less extracellular space and thus limited diffusion. Active inflammatory processes exhibit swelling or edematous changes in the cellular structure and likewise bring about a reduction in normal diffusion. Bowel imaging has also taken advantage of this. Initial examinations in patients with chronic inflammatory bowel diseases were able to differentiate healthy segments of the bowel and grade the severity of the disease on the basis of apparent diffusion coefficients (ADC) using DWI [34-36] ( $\bullet$ Fig. 4). It was also shown that adding diffusion-weighted sequences to the clinical examination protocol improved diagnostic certainty 

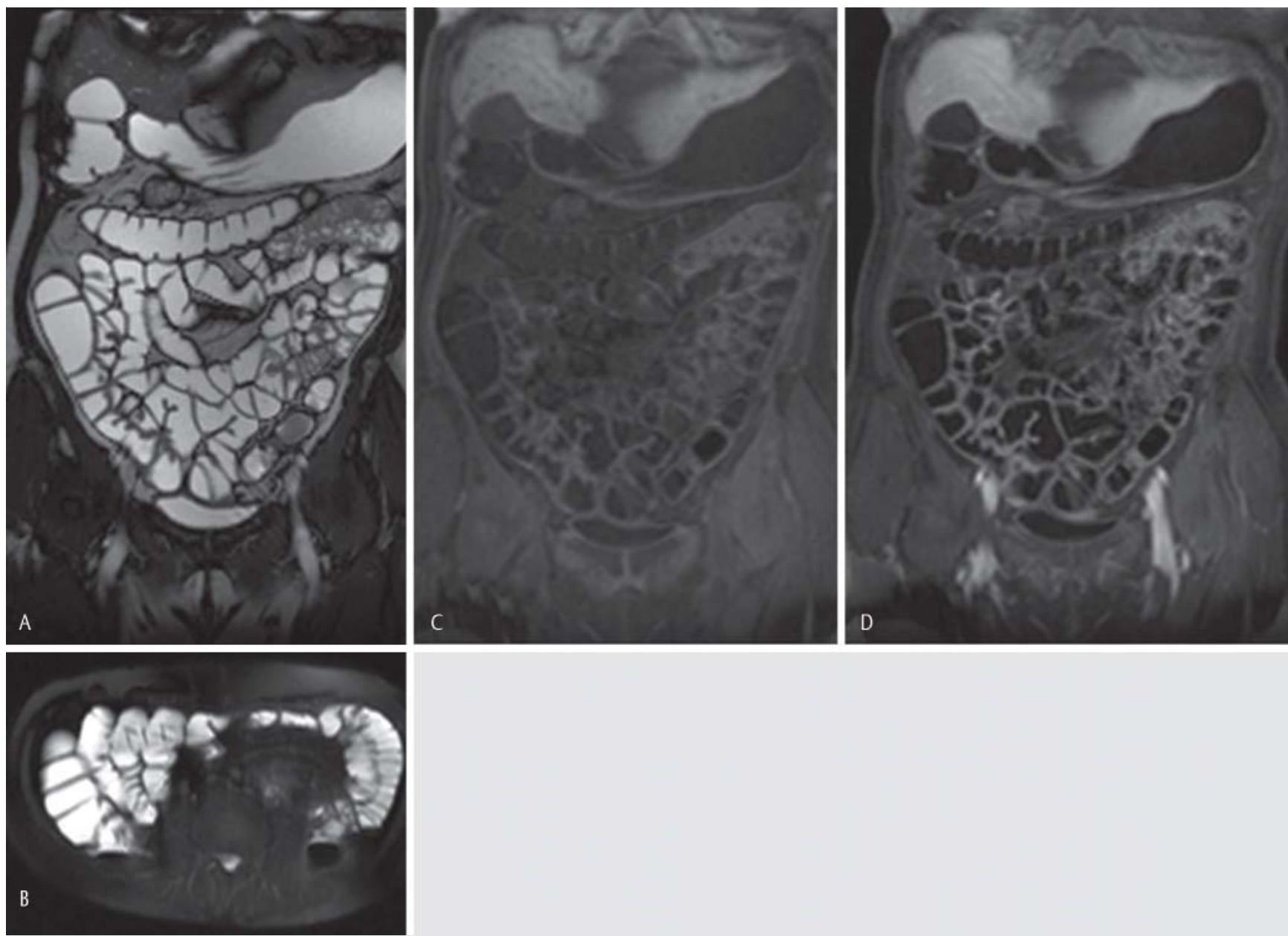

Fig. 3 A MR bowel protocol should include TrueFISP images A in coronal acquisition, T2 HASTE in axial or coronal acquisition B as well as dynamic fat saturated T1 weighted gradient echo sequences (VIBE, C, D) in coronal acquisition.

[37]. Both small and large intestine examinations were included in different clinical requests, reflecting the reality of clinical examinations. Diagnostic certainty was improved not only for inflammatory but also for tumorous lesions. Kilickesmez et al. showed that for examining the rectosigmoid colon of patients with inflammatory and neoplastic changes, DWI is able to differentiate normal from pathologically changed findings and could thus facilitate differentiating inflammation from tumor growth [38]. Schmid-Tannwald et al. discovered additional benefits of DWI for differentiating fistulas [39]. In their publication, these authors discussed the use of DWI on patients with contraindications for contrast medium (e.g. renal failure). An already published study as well as additional current studies compare the diagnostic accuracy of DWI with that of conventional contrast-enhanced bowel imaging in patients with chronic inflammatory bowel diseases [40]. As mentioned above, the fact that DWI makes it possible to omit the use of contrast medium would be beneficial not only in cases of kidney dysfunctions but also when repeated examinations are required in pediatric diagnostics.

Diffusion-weighted imaging allows the detection of accessory inflammatory or tumorous lesions, while increasing diagnostic certainty. Because it does not require the use of contrast medium, it can additionally be used particularly for examining patients with impaired kidney function and, because of the brief examination time involved, it can be used on pediatric patients, e.g. for evaluating response to therapy.

\section{MRI of bowel motility}

Because intestinal peristalsis is typically antegrade, chyme is propelled forward from the oral cavity to the rectum, being intensely mixed and processed along the way. Propulsion is facilitated by a contraction of the intestinal wall musculature [41]. The presence of an intestinal disease also affeing deeper layers of the intestinal wall can be manifested in this segment by a reduction or suspension of bowel motility. More recent studies have concerned themselves with these motility examinations, which are typically performed following the overview sequences and prior to administration of an antispasmotic. A T2-weighted real-time cine sequence can be employed to generate repetitive images, thereby representing bowel motility [42]. Comparison of the motility of individual intestinal segments can expose initial pathologies, since small intestinal segments in particular normally exhibit a regular, uniform contraction. Fröhlich et al. measured the changes in intestinal segment diameter over time during this process [26]. Current software solutions make it possible to quantify the reduced or sus- 

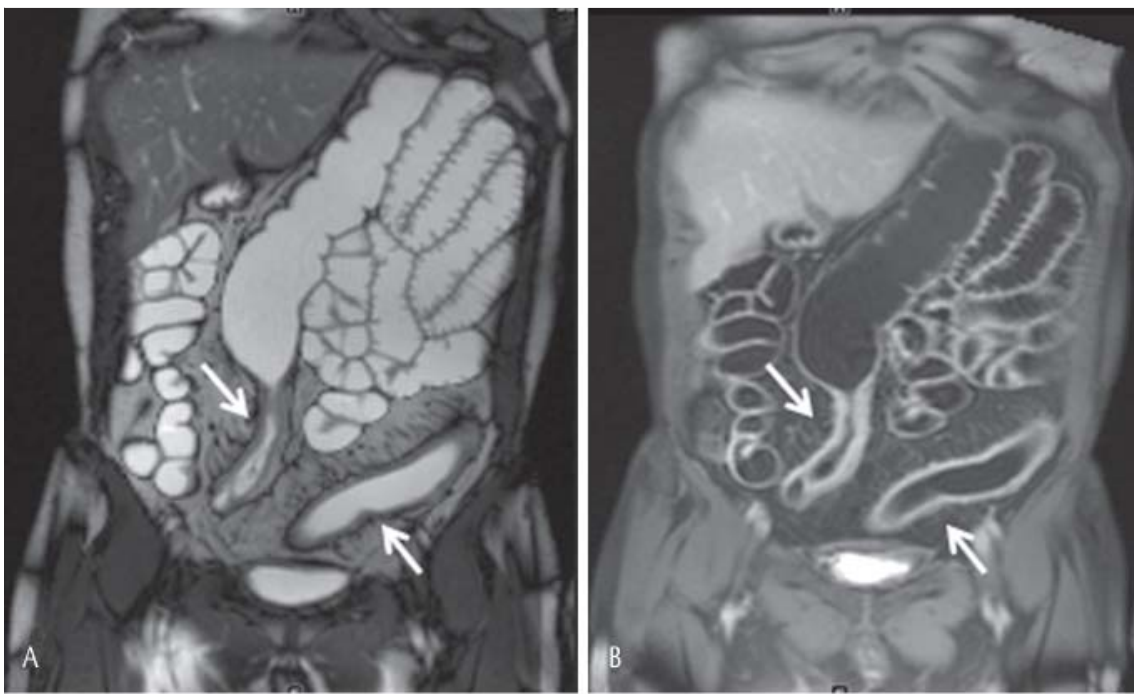

Fig. 454 year old patient with crohn `s disease after small bowel resection and ileoascendostomy. The patient was re-evaluated because of persisting diarrhea and inflammatory markers. T2-weighted images (A coronal TrueFISP) shows mural thickening in the region of ileoacendostomy as well as in the sigmoid colon with noticeable comb sign. Distinct contrast enhancement of inflamed bowel segments (B T1 TSE after contrast). DWI shows corresponding dissusion restriction with high signal in images acquired with $b=1000 C$ as well as signal reduction in $A D C$ (apparent diffusion coefficient) map $\mathbf{D}$.
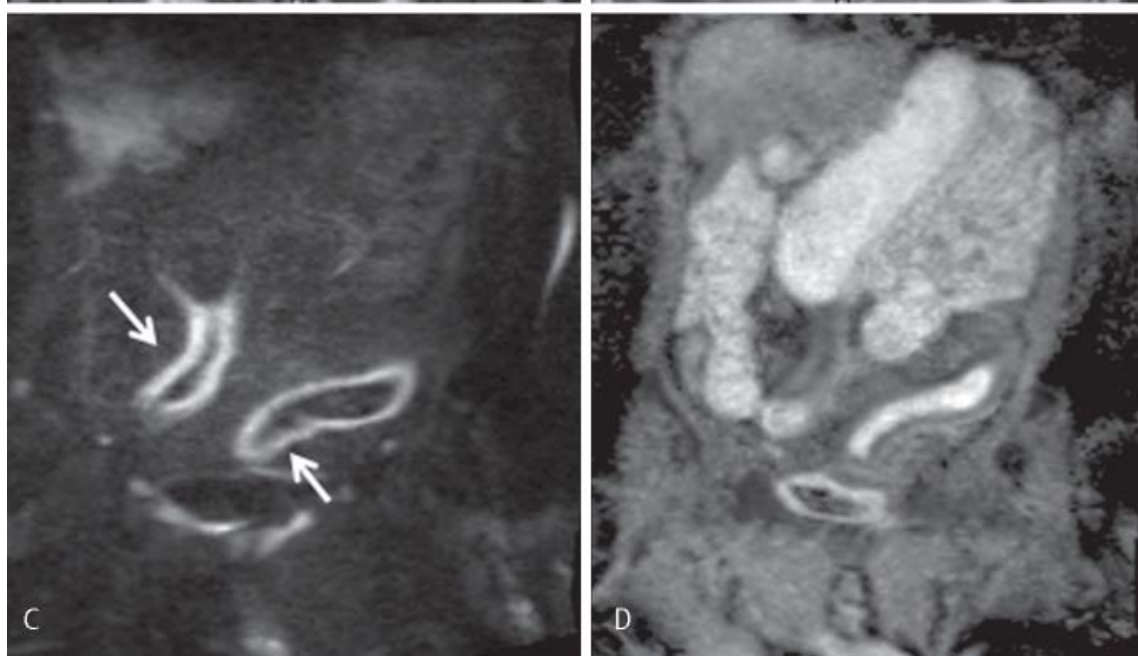

pended bowel motility, by measuring the luminal diameter and plot it along the temporal axis [43]. Software-based measurements have proven to be highly reliable, while allowing accurate and faster measurement. Hahnemann et al. show that compared to healthy subjects, Crohn's patients exhibit reduced or even suspended bowel motility in areas exhibiting acute inflammatory changes [44] ( $\bullet$ Fig.5). In their retrospective study, Menys et al. presented that the motility analysis can be used to differentiate normal intestinal segments as well as those with strictures or upstream from strictures [45]. In their analysis of Crohn's disease patients, Fröhlich et al. found that additionally analyzing bowel motility significantly boosted the detection of inflammatory lesions [46]. In this way not only were more inflammatory lesions detected in the affected patients compared to standard imaging, but also more patients were found to have lesions than with standard imaging. Bickelhaupt et al. showed impaired bowel motility to be closely correlated with the inflammation markers C-reactive protein and calprotectin [47].

MR imaging of bowel motility thus enables the detection of intestinal segments with inflammatory changes that were not detectable with standard imaging and are closely correlated with inflammatory activity measured with fecal and serological inflammation markers.

\section{Dynamic contrast enhanced (DCE) MRI}

Dynamic contrast-enhanced imaging has long been employed in oncology as a non-invasive method for evaluating tumor blood perfusion [48]. The potential of DCE-MRI as a biomarker for measuring antiangiogenic effects of cancer therapy can also be used in bowel imaging for evaluating the vascularization of intestinal segments and likewise for evaluating therapy response in both chronic inflammatory bowel diseases and in tumors of the bowel [49, 50]. In a study involving 18 patients, Oto et al. showed that combining DCE-MRI with new methods of diffusion-weighted imaging (DWI) provides a good quantitative measure for differentiating acutely inflammatory changes in the small intestine from normal intestinal segments [35]. However, DWI was even superior to DCE-MRT in this endeavor. Nevertheless, the combination of ADC values and perfusion parameters makes it possible to improve specificity. In their study of children with chronic inflammatory bowel disease, Alexopoulou et al. showed that the percentage-based enhancement of the intestinal wall was significantly higher in patients with abnormal C-reactive protein (CRP) values, i.e. with acute inflammation parameters, than in patients with normal CRP values [51]. DCE-MRI requires contrast medium administration within a strictly defined timeframe to allow the quantitative and qualitative analysis of time-signal- 

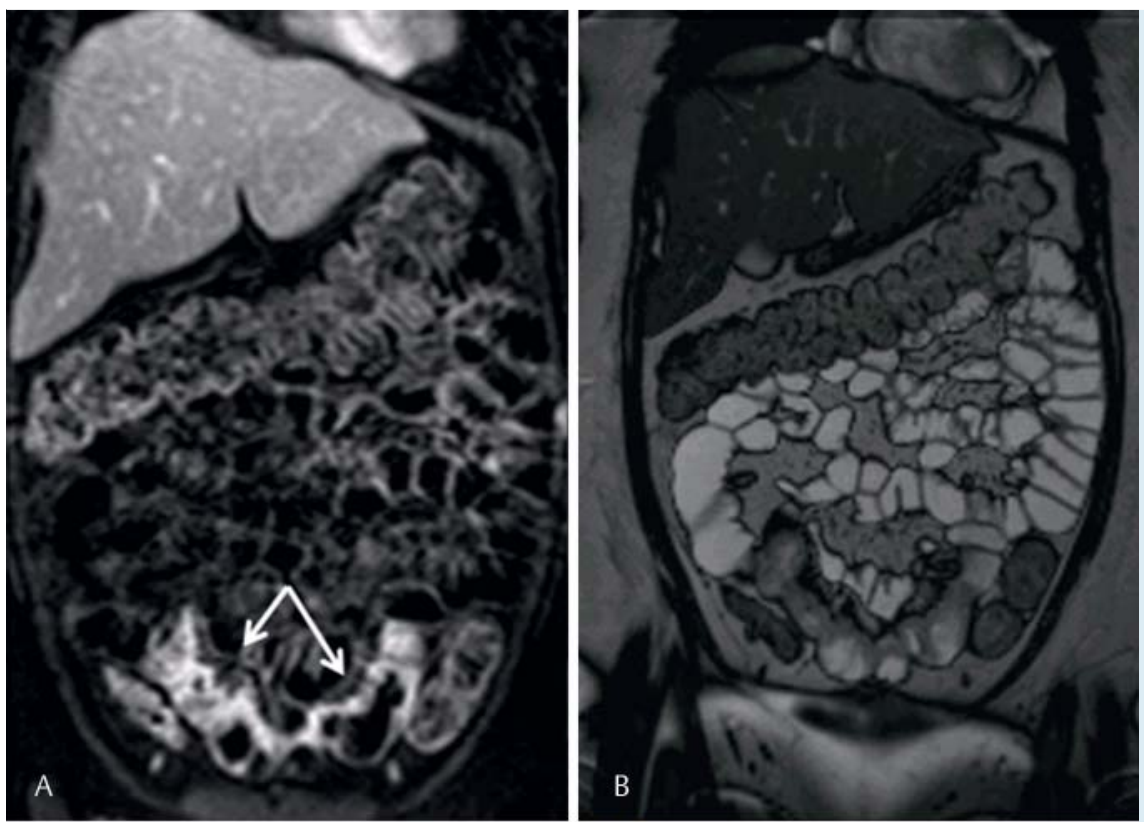

Fig. 515 year old female patient with known crohn's disease of the distal and terminal ileum. Distinct contrst uptake of the inflamed bowel segments are detectable on dynamic sequences $\mathbf{A}$. TrueFISP images additionally show marked mural thickening $\mathbf{B}$. Motility imaging (single $\mathrm{T} 2$ real-time image, $\mathbf{C}$ shows reduced motility of inflamed bowel parts. Software assisted colour coding shows this reduced motility in red (arrow, D).
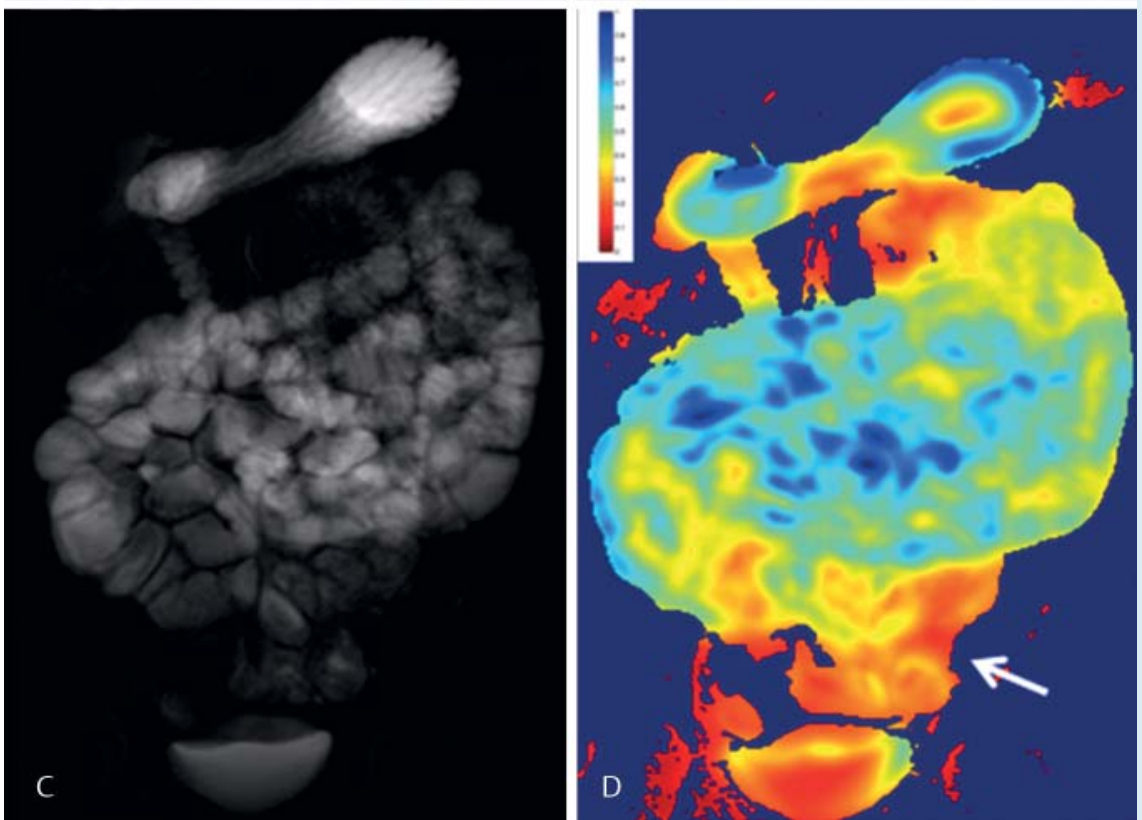

curves. In the future, 4-dimensional MR angiography sequences could also be beneficial here.

DCE-MRI is used particularly for evaluating acute inflammatory as well as tumorous processes and monitoring the response to therapy thereof. Its benefits appear to still be inferior to DWI in particular, and future studies are needed to establish its relative value.

\section{Hybrid imaging: PET/MR}

Positron emission tomography (PET) is a form of functional imaging. Using 18-F-fluorodeoxyglucose (18-F-FDG), this imaging technique takes advantage of glucose metabolism, since inflammatory and tumorous processes in the body have elevated glucose consumption and store 18-F-FDG at a greater rate. Combining computed tomography (CT) with PET/CT makes it possible to detect chronic inflammatory bowel diseases at higher sensitivity and specificity [52]. With its greater soft tissue contrast, MRI would appear to be ideal for being combined with the metabolic information of PET and thus achieving even better results than that of PET/CT as the first images from PET/MR examination highlight ( $\bullet$ Fig. 6 ). In addition, PET/MRI exposes patients to less radiation than $\mathrm{PET} / \mathrm{CT}$.

A study conducted by Lenze et al. already demonstrated that the combination of individual examinations yielded better results than 18-F-FDG PET/CT, MR enteroclysis and transabdominal ultrasound alone in the detection and differentiation of acute inflammatory changes over fibrotic strictures in Crohn's disease patients alone [53]. It is expedient for PET and MR data to be acquired simultaneously when PET/MRI is employed. This method is particularly relevant if the position of the intestinal loops shows drastic change within a brief period and offers in combination the highest accuracy for detecting pathologies. The nuclear medicine component of PET/MRI cannot rely just on 18-FFDG as tracer. It is conceivable that additional, radiotracer- 

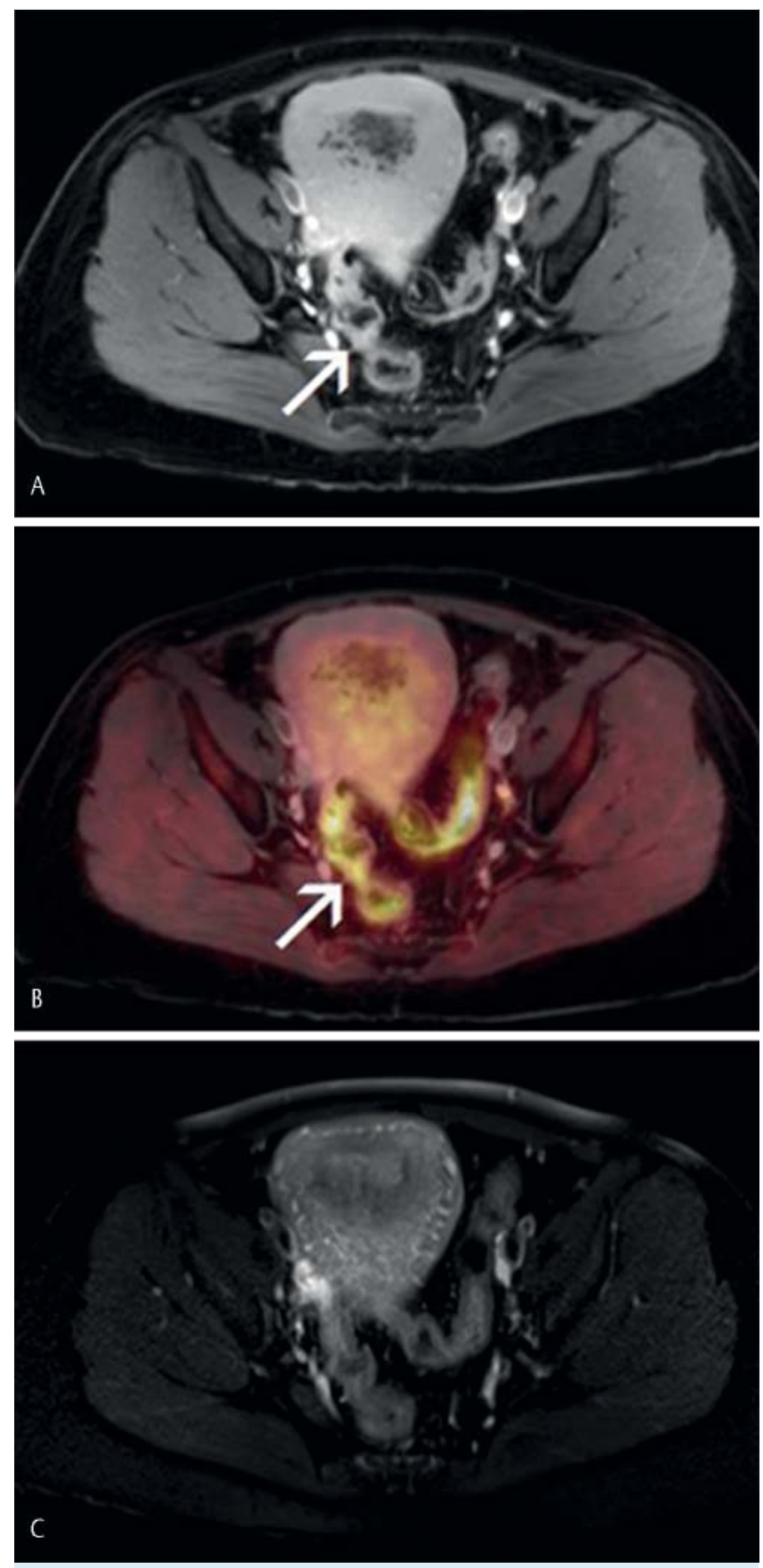

Fig. 628 year old female patient with initial diagnosis of breast cancer and whole-body PET MR imaging with 18F-FDG for staging and known crohn 's disease. Contrast enhancement and slight wall thickening can be seen in the rectosigmoid colon (A T1w FLASH after contrast, arrow). PET images show enhanced glucose uptake $\mathbf{B}$. T2-weighted images of non-distended, non-prepared bowel show only slight mural edema (C T2 HASTE fat saturated). Imaging patients with inflammatory bowel disease therefore seems to be promising.

labelled cells or receptors can be used in the future for both inflammatory changes and tumorous processes.

Future studies on both patients with chronic inflammatory bowel diseases and patients with gastrointestinal tumors are still needed to shed light on the current value of PET/ MRI. In addition to the conventional morphological sequences, the other innovative sequences such as DWI and DCE-
MRI will be used as functional imaging modalities among these patients.

\section{Conclusions}

\section{$\nabla$}

T2-weighted sequences both with and without fat saturation and T1-weighted fat saturated sequences prior to and following intravenous injection of a gadolinium-based contrast medium constitute basic diagnostic approaches in the magnetic resonance imaging of the bowel. Newer MR applications, such as diffusion-weighted imaging or contrast-enhanced dynamic sequences supply additional information in the diagnosing of chronic inflammatory bowel diseases as well as bowel tumors and should thus be integrated into the regular sequence protocol. Additional new imaging modalities such as motility imaging or the combination with PET in the form of PET/MRI must continue to be evaluated in the future and are very likely to become valuable tools in diagnosing chronic inflammatory bowel diseases. These innovative imaging modalities could provide deeper understanding of the underlying diseases and allow the possibility of functional, non-invasive therapy monitoring.

\section{References}

1 Luboldt W, Debatin JF. Virtual endoscopic colonography based on 3D MRI. Abdom Imaging 1998; 23: 568 - 572

2 Luboldt W, Steiner P, Bauerfeind P et al. Detection of mass lesions with MR colonography: preliminary report. Radiology 1998; 207: 59-65

3 Platzer IA, Neubauer H, Beer M. Mittels Hydro-Sonografie und HydroMRT juvenilen Kolonpolypen auf die Spur kommen. Fortschr Röntgenstr 2012; 183: 744-745

4 van der Paardt MP, Stoker J. Magnetic Resonance Colonography for Screening and Diagnosis of Colorectal Cancer. Magnetic Resonance Imaging Clinics of North America 2014; 22: 67-83

5 Lauenstein TC, Rühm SG, Debatin JF. Aktuelle Standards der MR-Kolonographie. Fortschr Röntgenstr 2003; 175: 334-341

6 Graser A, Melzer A, Lindner E et al. Magnetic resonance colonography for the detection of colorectal neoplasia in asymptomatic adults. Gastroenterology 2013; 144: 743 - 750 e742

7 Hartmann D, Bassler B, Schilling D et al. Incomplete Conventional Colonoscopy: Magnetic Resonance Colonography in the Evaluation of the Proximal Colon. Endoscopy 2005; 37: 816-820

8 Kovanlikaya A, Watson E, Hayward J et al. Magnetic resonance enterography and wireless capsule endoscopy in the evaluation of patients with inflammatory bowel disease. Clinical imaging 2013; 37: 77-82

9 Anupindi SA, Terreblanche O, Courtier J. Magnetic Resonance Enterography: Inflammatory Bowel Disease and Beyond. Magnetic Resonance Imaging Clinics of North America 2013; 21: 731 - 750

10 Schleder S, Pawlik M, Wiggermann P et al. Interobserver Agreement in MR Enterography for Diagnostic Assessment in Patients with Crohn's Disease. Fortschr Röntgenstr 2013; 185: $992-997$

11 Wessling J, Fischbach R, Borchert A et al. Detection of colorectal polyps: comparison of multi-detector row CT and MR colonography in a colon phantom. Radiology 2006; 241: 125-131

12 Röttgen R, Herzog H, Bogen P et al. MR colonoscopy at 3.0 T: comparison with $1.5 \mathrm{~T}$ in vivo and a colon model. Clinical imaging 2006; 30 : $248-253$

13 Fiorino G, Bonifacio C, Padrenostro $M$ et al. Comparison Between 1.5 and 3.0 Tesla Magnetic Resonance Enterography for the Assessment of Disease Activity and Complications in Ileo-Colonic Crohn's Disease. Dig Dis Sci 2013; 58: 3246-3255

14 Borthne A, Abdelnoor M, Storaas T et al. Osmolarity: a decisive parameter of bowel agents in intestinal magnetic resonance imaging. Eur Radiol 2006; 16: 1331 -1336

15 Ajaj W, Goehde S, Schneemann $H$ et al. Oral contrast agents for small bowel MRI: comparison of different additives to optimize bowel distension. Eur Radiol 2004; 14: 458-464 
16 Kinner S, Kuehle C, Herbig S et al. MRI of the small bowel: can sufficient bowel distension be achieved with small volumes of oral contrast? Eur Radiol 2008; 18: 2542 - 2548

17 Laghi A, Paolantonio P, Iafrate F et al. Oral Contrast Agents for Magnetic Resonance Imaging of the Bowel. Topics in Magnetic Resonance Imaging 2002; 13: 389-396

18 McKenna DA, Roche CJ, Murphy JMP et al. Polyethylene glycol solution as an oral contrast agent for MRI of the small bowel in a patient population. Clinical radiology 2006; 61: 966 -970

19 Masselli G, Casciani E, Polettini E et al. Comparison of MR enteroclysis with MR enterography and conventional enteroclysis in patients with Crohn's disease. Eur Radiol 2008; 18: 438 - 447

20 Negaard A, Paulsen V, Sandvik L et al. A prospective randomized comparison between two MRI studies of the small bowel in Crohn's disease, the oral contrast method and MR enteroclysis. Eur Radiol 2007; 17: $2294-2301$

21 Schreyer AG, Geissler A, Albrich $\mathrm{H}$ et al. Abdominal MRI after enteroclysis or with oral contrast in patients with suspected or proven Crohn's disease. Clinical Gastroenterology and Hepatology 2: 491-497

22 Arrivé L, El Mouhadi S. MR Enterography versus MR Enteroclysis. Radiology 2013; 266: 688-688

23 Lauenstein TC, Herborn CU, Vogt FM et al. Dark lumen MR-Colonography: initial experience. Fortschr Röntgenstr 2001; 173: 785 - 789

24 Friedrich C, Fajfar A, Pawlik M et al. Magnetic resonance enterography with and without biphasic contrast agent enema compared to conventional ileocolonoscopy in patients with Crohn's disease. Inflammatory Bowel Diseases 2012; 18: 1842 -1848. DOI: 1810.1002/ibd.22843

25 Santillan CS. MR Imaging Techniques of the Bowel. Magnetic Resonance Imaging Clinics of North America 2014; 22: 1-11

26 Froehlich J, Daenzer M, Weymarn C et al. Aperistaltic effect of hyoscine $\mathrm{N}$-butylbromide versus glucagon on the small bowel assessed by magnetic resonance imaging. Eur Radiol 2009; 19: 1387-1393

27 Gutzeit A, Binkert C, Koh D-M et al. Evaluation of the anti-peristaltic effect of glucagon and hyoscine on the small bowel: comparison of intravenous and intramuscular drug administration. Eur Radiol 2012; 22: $1186-1194$

28 Schleder S, Dendl LM, Pawlik M et al. Sequenz-Evaluation für die MREnterografie für Patienten mit Morbus Crohn. Fortschr Röntgenst 2013: $185: 440-445$

29 Ajaj W, Rühm SG, Papanikolaou N et al. Dark Lumen MR Colonography: Can High Spatial Resolution VIBE Imaging Improve the Detection of Colorectal Masses? Fortschr Röntgenstr 2006; 178: 1073-1078

30 Minematsu K, Li L, Fisher $M$ et al. Diffusion-weighted magnetic resonance imaging: rapid and quantitative detection of focal brain ischemia. Neurology 1992; 42: 235-240

31 Hartmann M, Heiland S, Sartor K. Funktionelle MR-Verfahren in der Diagnostik intraaxialer Hirntumoren: Perfusions- und Diffusions-Bildgebung. Fortschr Röntgenstr 2002; 174: 955 -964

32 Bohlscheid A, Nuss D, Lieser S et al. Tumorsuche mittels kernspintomografischer Diffusionsbildgebung. Fortschr Röntgenstr 2008; 180 $302-309$

33 Wilhelm T, Stieltjes B, Schlemmer HP. Whole-Body-MR-Diffusion Weighted Imaging in Oncology. Fortschr Röntgenstr 2013; 185: 950 958

34 Buisson A, Joubert A, Montoriol PF et al. Diffusion-weighted magnetic resonance imaging for detecting and assessing ileal inflammation in Crohn's disease. Alimentary Pharmacology \& Therapeutics 2013; 37: $537-545$

35 Oto A, Kayhan A, Williams JTB et al. Active Crohn's Disease in the small bowel: Evaluation by diffusion weighted imaging and quantitative dynamic contrast enhanced MR imaging. Journal of Magnetic Resonance Imaging 2011; 33: 615-624
36 Oussalah A, Laurent V, Bruot 0 et al. Diffusion-weighted magnetic resonance without bowel preparation for detecting colonic inflammation in inflammatory bowel disease. Gut 2010; 59: 1056-1065

37 Kinner S, Blex S, Maderwald S et al. Addition of diffusion-weighted imaging can improve diagnostic confidence in bowel MRI. Clinical radiology 2013

38 Kilickesmez O, Atilla S, Soylu A et al. Diffusion-Weighted Imaging of the Rectosigmoid Colon: Preliminary Findings. Journal of Computer Assisted Tomography 2009; 33: 863-866. DOI: 810.1097| RCT.1090b1013e31819a31860f31813

39 Schmid-Tannwald C, Agrawal G, Dahi F et al. Diffusion-weighted MRI: Role in detecting abdominopelvic internal fistulas and sinus tracts. Journal of Magnetic Resonance Imaging 2012; 35: 125 - 131

40 Neubauer H, Pabst T, Dick A et al. Small-bowel MRI in children and young adults with Crohn disease: retrospective head-to-head comparison of contrast-enhanced and diffusion-weighted MRI. Pediatr Radiol 2013; 43: $103-114$

41 Kunze WA, Furness JB. The enteric nervous system and regulation of intestinal motility. Annual review of physiology 1999; 61: 117-142

42 Menys A, Taylor SA, Emmanuel A et al. Global Small Bowel Motility: Assessment with Dynamic MR Imaging. Radiology 2013; 269: 443-450

43 Bickelhaupt S, Froehlich JM, Cattin $R$ et al. Software-assisted quantitative analysis of small bowel motility compared to manual measurements. Clinical radiology 2014

44 Hahnemann ML, Nensa F, Kinner S et al. Motility mapping as evaluation tool for bowel motility: Initial results on the development of an automated color-coding algorithm in cine MRI. J Magn Reson Imaging 2014; DOI: 10.1002/jmri.24557. [Epub ahead of print]

45 Menys A, Helbren E, Makanyanga J et al. Small bowel strictures in Crohn's disease: a quantitative investigation of intestinal motility using MR enterography. Neurogastroenterology \& Motility 2013; 25: $967-\mathrm{e} 775$

46 Froehlich J, Waldherr C, Stoupis C et al. MR motility imaging in Crohn's disease improves lesion detection compared with standard MR imaging. Eur Radiol 2010; 20: 1945 - 1951

47 Bickelhaupt S, Pazahr S, Chuck N et al. Crohn's disease: small bowel motility impairment correlates with inflammatory-related markers C-reactive protein and calprotectin. Neurogastroenterology \& Motility 2013; 25: 467-e363

48 Hylton N. Dynamic Contrast-Enhanced Magnetic Resonance Imaging As an Imaging Biomarker. Journal of Clinical Oncology 2006; 24: $3293-3298$

49 Oto A, Fan X, Mustafi D et al. Quantitative Analysis of Dynamic Contrast Enhanced MRI for Assessment of Bowel Inflammation in Crohn's Disease: Pilot Study. Academic radiology 2009; 16: $1223-1230$

50 Tang JS, Choy G, Bernardo $M$ et al. Dynamic Contrast-Enhanced Magnetic Resonance Imaging in the Assessment of Early Response to Tumor Necrosis Factor Alpha in a Colon Carcinoma Model. Investigative Radiology 2006; 41: 691-696. DOI: 610.1097/1001. rli.0000233882.0000283800.fb

51 Alexopoulou E, Roma E, Loggitsi D et al. Magnetic resonance imaging of the small bowel in children with idiopathic inflammatory bowel disease: evaluation of disease activity. Pediatr Radiol 2009; 39: 791 - 797

52 Berthold $L D$, Steiner D, Scholz D et al. Imaging of Chronic Inflammatory Bowel Disease with 18F-FDG PET in Children and Adolescents. Klin Padiatr 2013; 225: $212-217$

53 Lenze F, Wessling J, Bremer J et al. Detection and differentiation of inflammatory versus fibromatous Crohn's disease strictures: Prospective comparison of 18F-FDG-PET/CT, MR-enteroclysis, and transabdominal ultrasound versus endoscopic/histologic evaluation. Inflammatory Bowel Diseases 2012; 18: 2252 -2260. DOI: 2210.1002/ ibd.22930 